\title{
Prevalência de sintomas depressivos e ansiosos em pacientes com dor crônica
}

\author{
Prevalence of depressive and anxiety symptoms in patients with chronic pain \\ Ricardo Cardoso Pinheiro', Ricardo Riyoiti Uchida², Lígia Andrade da Silva Telles Mathias³, \\ Marcelo Vaz Perez $z^{4}$ Quirino Cordeiro ${ }^{5}$
}

\section{RESUMO}

Objetivos: $O$ presente estudo objetivou avaliar a prevalência de sintomas ansiosos e depressivos e relacioná-los com os diferentes tipos e magnitudes de dor crônica; também objetivou caracterizar a população de casos novos agendados para atendimento no ambulatório de dor crônica, no serviço onde o estudo foi realizado. Métodos: Estudo de corte transversal, realizado em um ambulatório docente-assistencial para tratamento de dor crônica, realizado entre maio de 2012 e abril de 2013, com 125 pacientes. Instrumentos utilizados: Questionário sociodemográfico, Escala Hospitalar de Ansiedade e Depressão (HAD) e Escala Visual Numérica (EVN) para aferir a intensidade de dor. Resultados: Dor intensa foi referida por $64 \%$ ( $n=$ $80)$ dos pacientes. Ansiedade atingiu $65 \%(N=82)$ dos pacientes e a depressão, 48\% ( $N=60)$. Houve correlação significante entre os mais altos escores de ansiedade $(p<0,001)$ e depressão $(p<0,001)$ com a intensidade de dor. A correlação entre intensidade de dor e sintomas ansiosos e depressivos foi positiva para dor crônica neuropática e mista. Os fatores sociodemográficos associados à intensidade de dor crônica foram: renda e religião para depressão, e

\section{Palavras-chave}

Dor, humor, ansiedade, depressão. tempo de dor para ansiedade. Conclusão: $O$ estudo mostrou elevada prevalência de sintomas depressivos e ansiosos em pacientes com dor crônica, assim como relação significante desses sintomas psiquiátricos com alguns tipos e intensidade de dor.

\section{ABSTRACT}

Objectives: The present study aimed to evaluate the prevalence of anxiety and depressive symptoms and their association with different types and intensity of chronic pain. The investigation also characterized the population profile of new cases scheduled for appointments at the pain outpatient clinic, where the study was carried out. Methods: Cross-sectional study, carried out at a chronic pain treatment outpatient-teaching clinic, between May 2012 and April 2013, with 125 patients. The following instruments were used: Sociodemographic questionnaire, Hospital Anxiety and Depression Scale (HADS), and Visual Numeric Scale (VNS) to assess the intensity of pain. Results: Intense pain was reported by $64 \%(n=80)$ of patients. With a cut-off of 8 points for HAD scale, anxiety affected $65 \%(\mathrm{~N}=82)$ of patients, and depression affected

1 Faculdade de Ciências Médicas da Santa Casa de São Paulo (FCMSCSP).

2 FCMSCSP, Departamento de Psiquiatria.

3 FCMSCSP, Departamento de Anestesiologia.

4 Irmandade da Santa Casa de Misericórdia de São Paulo (ISCMSP), Serviço de Anestesiologia.

5 FCMSCSP, Departamento de Psiquiatria.

3/10/2014

DOI: 10.1590/0047-2085000000028
Endereço para correspondência: Quirino Cordeiro Centro de Atenção Integrada à Saúde Mental (CAISM), Santa Casa de Misericórdia de São Paulo Rua Major Maragliano, 241 04017-030 - São Paulo, SP, Brasil

E-mail:qcordeiro@yahoo.com 


\section{Keywords}

Pain, humor, anxiety, depression.
48\% ( $N=60)$. We found a significant correlation between higher scores of anxiety $(p<0.001)$ and depression $(p<0.001)$ with pain intensity. The correlation between pain intensity, anxiety and depressive symptoms was positive for neuropathic pain as well as for mixed chronic pain. Sociodemographic factors associated to chronic pain intensity were income and religion for depression, and time living with pain for anxiety. Conclusion: The present study showed high prevalence of depressive and anxiety symptoms in patients with chronic pain. There was also significant association of these psychiatric symptoms with some types and intensity of pain.

\section{INTRODUÇÃO}

A associação entre dor e transtornos mentais é bastante referida na literatura médica. Do ponto de vista psiquiátrico, tal associação pode ocorrer quando o transtorno mental: (1) provoca o aparecimento de condições físicas que causam dor; (2) piora a condição dolorosa, evento comum nos quadros de depressão; (3) é secundário ao aparecimento de dor física, propiciando condições para que o transtorno psiquiátrico aflore; ou (4) pode apresentar como queixa a dor'.

A dor é um dos temas que atualmente faz parte do universo da psiquiatria, propondo desafios pelas condições científicas e socioculturais que permeiam o estabelecimento da comorbidade entre síndromes dolorosas e transtornos psiquiátricos. No entanto, a fisiopatologia comum aos quadros álgicos e psiquiátricos não pode ser inferida diretamente pela ação terapêutica de medicamentos, os quais podem ter efeitos positivos em ambos os quadros. Considerando-se que os medicamentos têm ações diversas sobre as doenças, há casos em que a fisiopatologia não é a mesma, apesar de a resposta terapêutica ser positiva para ambos os quadros.

A dor não pode ser avaliada diretamente e ela é sempre inferida com base nas informações do paciente. Além dos fatores subjetivos e perceptivos, a complexidade desse fenômeno envolve fatores culturais, sociais, contingenciais e fisiológicos, que respondem pela diversidade de respostas singulares de pacientes que buscam alívio para o seu sofrimento. Disso decorrem as dificuldades de delimitação etiológica, nosográfica e diagnóstica da dor².

A classificação fisiopatológica ${ }^{3}$ da dor envolve três categorias, a saber: dor neuropática, com lesão primária ou disfunção do sistema nervoso central e/ou do sistema nervoso periférico; dor nociceptiva ou somática, caracterizada pela ativação contínua dos nociceptores por lesão tecidual; dor mista, em que ambos os componentes estão presentes, sendo este o tipo de dor mais frequente.

A percepção da condição dolorosa pode ser fortemente alterada conforme o valor atribuído a ela na situação vivida. Assim, os fatores sociodemográficos associados às diversas condições de dor crônica têm sido: gênero feminino, idosos, baixa condição socioeconômica, antecedentes culturais e geográficos, situação de desemprego e fatores ocupacionais, história de abuso ou de violência interpessoal ${ }^{4}$.

Uma revisão sistemática ${ }^{5}$ sobre a prevalência de dor crônica na população geral de países desenvolvidos aponta média de 35,5\%, variando de $11,5 \%$ a 55,5\%, sendo de característica intensa em $11 \%$ dos adultos, considerando-se os critérios estabelecidos pela International Association for the Study of Pain (IASP): ausência de base biológica aparente e duração por três meses ou mais, ou seja, além do período esperado para a cicatrização tecidual normal. No Brasil, a dor crônica é estimada entre 30\% e 40\% da população ${ }^{6}$, mas não há registro de estudos epidemiológicos com amostra populacional representativa do país?.

A associação entre dor crônica e transtornos psiquiátricos é reportada em vários estudos ${ }^{8}$, porém as características e magnitudes de tal associação não são bem conhecidas. Entre os transtornos psiquiátricos, a depressão maior foi mais amplamente estudada e sua coocorrência foi mais bem estabelecida em pacientes com dor crônica ${ }^{8-10}$. Estudos de prevalência de comorbidades psiquiátricas associadas à dor crônica referem em primeiro lugar os transtornos de humor, entre os quais os transtornos depressivos alcançam porcentagens entre 30\% e $87 \%$ dos casos; a depressão maior, de $8 \%$ a 50\% e a distimia está associada em mais de $75 \%$ das situações. Entre os transtornos ansiosos, que chegam a 50\% dos casos, os mais frequentes são: transtorno do pânico, transtorno de ansiedade generalizada e transtorno de estresse pós-traumático. Como sintoma, a ansiedade está presente em 56\% dos casos, e em menos de 10\% a ansiedade desenvolveu-se após o quadro doloroso.

Diante desse panorama, o presente estudo teve como objetivo: avaliar a prevalência de sintomas ansiosos e depressivos dos pacientes com dor crônica e relacioná-la com os diferentes tipos e magnitudes de dor. Além disso, a investigação objetivou caracterizar a população de casos novos atendidos em um serviço de referência no tratamento de dor crônica na cidade de São Paulo, com base em parâmetros clínicos relativos à dor e aos sintomas depressivos e ansiosos e, ainda, a parâmetros sociodemográficos.

\section{MÉTODOS}

\section{Aspectos éticos}

O estudo foi aprovado pelo Comitê de Ética em Pesquisa em seres humanos da instituição onde o trabalho foi realizado, sob o no 007/12-PB. 
Os participantes foram solicitados a assinar o Termo de Consentimento Livre e Esclarecido, após os devidos esclarecimentos sobre o estudo e antes da coleta dos dados.

\section{Casuística}

Tratou-se de estudo de corte transversal da população atendida em ambulatório de tratamento de dor crônica da cidade de São Paulo, conduzido com 125 pacientes novos, com dados coletados no período de maio de 2012 a abril de 2013.

O tamanho da amostra foi baseado no uso de regressão linear múltipla, tendo a intensidade de dor como variável dependente (resposta) e 11 preditores relativos à Escala Hospitalar de Ansiedade e Depressão (HAD), intensidade de dor, tempo de dor, tipos de dor e variáveis sociodemográficas (faixa etária, sexo, solidão, escolaridade, atividade laboral, religião, renda familiar). Utilizou-se o coeficiente de determinação $r^{2}=0,19$, com base nos dados do trabalho de Brasil e Pondé ${ }^{11}$. Erro tipo I e tipo II fixados em 5\%.

Critérios de inclusão: pacientes novos agendados para atendimento no grupo de dor crônica, com idade igual ou superior a 18 anos e alfabetizados. Critérios de exclusão: analfabetismo, não concordância quanto à participação na pesquisa e paciente reagendado ou em retorno.

\section{Instrumentos e procedimentos para coleta de dados}

Foram utilizados os seguintes instrumentos para a coleta de dados:

1. Questionário de dados sociodemográficos e clínicos, incluindo nome, idade, sexo, encaminhamento, estado civil, filhos, com quem mora, escolaridade, formação profissional, dados funcionais, faixa de renda familiar, religião, diagnóstico de dor, tempo de dor, outros ambulatórios de dor frequentados anteriormente, uso de medicação psicotrópica atual e Escala Visual Numérica de Dor (EVN) para intensidade da dor, variando de 0 a 10, sendo 0 sem dor e 10 a máxima dor.

2. Escala Hospitalar de Ansiedade e Depressão (HAD). Este instrumento consiste em 14 itens, sendo sete relativos a sintomas de ansiedade e sete relativos à depressão, devendo ser respondido com base na percepção dos sintomas na semana anterior ao preenchimento da escala. Cada item é formulado no modelo Likert, com quatro possibilidades que variam em pontuação de 0 a 3, visando medir a concordância do entrevistado com as afirmações propostas que buscam saber a intensidade e/ou frequência dos sintomas, ou a situação deles em relação a momentos anteriores da vida.

O questionário e as escalas EVN e HAD foram respondidos individualmente pelos pacientes e preenchidos por um profissional da área médica (anestesiologista ou psiquiatra) previamente treinado na coleta de dados, sem interferir na qualidade das respostas. O diagnóstico de dor foi feito durante a anamnese e exame pelo profissional especialista em dor, anteriormente à coleta de dados.
A escolha da HAD deu-se por sua validação anterior pela equipe do serviço de dor, o mesmo ambulatório em que foi realizada a presente pesquisa, denotando ser um instrumento de fácil aplicação e bem indicado para triagem de transtornos psiquiátricos, uma vez que exclui a presença de sintomas somáticos que poderiam distorcer os resultados quanto aos sintomas ansiosos e depressivos. Já a utilização da EVN deu-se pelo consenso da prática clínica na verificação na magnitude de dor.

Quanto aos tipos de dor, foi utilizada a classificação fisiopatológica da dor, segundo o Protocolo Clínico de Diretrizes Terapêuticas da Dor Crônica do Ministério da Saúde³, trabaIhando-se com três categorias: dor nociceptiva, dor neuropática e dor mista. Os dados com os diagnósticos originais dos 125 participantes foram enviados em planilhas para dois especialistas em dor, os quais foram convidados a classificar os diagnósticos em um dos três tipos. Houve concordância inicial em 83\% da amostra, e os 17\% discordantes mereceram uma nova discussão entre os especialistas, após o que a concordância resultou em 100\%.

\section{Análise dos dados}

A descrição estatística dos dados foi elaborada por meio do cálculo de frequências absolutas e relativas, medidas de tendência central (média e mediana) e dispersão (desvio-padrão, mínimo e máximo).

Para a análise estatística, foi realizada a correlação de Spearman, dado que as variáveis não apresentaram distribuição normal (teste Kolmogorov-Smirnov), e também a análise de regressão linear univariada para verificar a intensidade da dor crônica em relação aos escores de ansiedade e depressão. Para verificar a associação entre as variáveis independentes com o desfecho dor crônica, foram utilizados o teste do qui-quadrado e modelos de regressão logística univariados e múltiplos. As variáveis que apresentaram valor de $p<20 \%$ na análise univariada foram testadas no modelo múltiplo e com relevância clínica. Para a significância estatística, foi assumido um nível descritivo de 5\%. Os dados foram digitados em Excel e analisados em SPSS (Statistical Package for the Social Sciences), versão 17.0 para Windows.

\section{RESULTADOS}

A amostra do estudo foi composta por 125 pacientes. As características sociodemográficas podem ser observadas na tabela 1.

Em relação à renda, $80 \%$ ganhavam até quatro salários-mínimos, sendo 40\% até dois salários-mínimos e 40\% entre três e quatro salários-mínimos. Apenas 19,2\% do pacientes recebiam acima de cinco salários-mínimos. A maioria não estava em atividade laboral (69,6\%) e 91\% estavam inscritos no seguro social - INSS. 
Tabela 1. Características sociodemográficas

\begin{tabular}{|c|c|c|}
\hline Variável & $\mathrm{n}$ & $(\%)$ \\
\hline \multicolumn{3}{|l|}{ Sexo } \\
\hline Masculino & 42 & $(33,6)$ \\
\hline Feminino & 83 & $(66,4)$ \\
\hline \multicolumn{3}{|l|}{ Idade em anos (tercis) } \\
\hline 19 a 47 & 40 & $(32,0)$ \\
\hline 48 a 62 & 42 & $(33,6)$ \\
\hline 63 a 92 & 43 & $(34,4)$ \\
\hline \multicolumn{3}{|l|}{ Escolaridade } \\
\hline Fundamental incompleto & 46 & $(36,8)$ \\
\hline Fundamental - completo ou médio incompleto & 29 & $(23,2)$ \\
\hline Médio completo e superior & 50 & $(40,0)$ \\
\hline \multicolumn{3}{|l|}{ Religião } \\
\hline Católico & 75 & $(60,0)$ \\
\hline Evangélico & 32 & $(25,6)$ \\
\hline Outras & 10 & $(8,0)$ \\
\hline Sem religião & 8 & $(6,4)$ \\
\hline \multicolumn{3}{|l|}{ Estado civil } \\
\hline Solteiro & 23 & $(18,4)$ \\
\hline Casado/união estável & 74 & $(59,2)$ \\
\hline Separado/divorciado & 11 & $(8,8)$ \\
\hline Viúvo & 17 & $(13,6)$ \\
\hline \multicolumn{3}{|l|}{ Número de filhos } \\
\hline Nenhum & 21 & $(16,8)$ \\
\hline 1 a 2 & 61 & $(48,8)$ \\
\hline 3 e mais & 43 & $(34,4)$ \\
\hline \multicolumn{3}{|l|}{ Mora com } \\
\hline Sozinho & 13 & $(10,4)$ \\
\hline Cônjuge & 74 & $(59,2)$ \\
\hline Outros parentes ou acompanhantes & 38 & $(30,4)$ \\
\hline Total & 125 & $(100,0)$ \\
\hline
\end{tabular}

Quanto aos dados clínicos, a dor mista representou a maioria dos casos (52\%; $N=65)$, seguida pela dor neuropática, com 31,2\% dos casos ( $N=39)$. Em relação ao tempo de dor, observou-se que os quartis foram amplos, pois sua distribuição apresentou variação de 3 a 480 meses (ou 40 anos); a média de tempo de dor foi 76,4 meses (6,3 anos) ( $d p=81,9)$, mediana de 48 ( 4 anos). A dor intensa, graduada entre 8 e 10 pontos na EVN, foi referida por $64 \%(n=80)$ dos pacientes. A escala de dor apresentou uma média de 7,6 pontos ( $\mathrm{dp}=$ 2,5), mediana de 8 , valor mínimo de 0 e máximo de 10. De acordo com a escala HAD, a ansiedade possível ou provável atingiu 65\% ( $N=82$ ) dos pacientes e a depressão possível ou provável esteve presente em $48 \%(\mathrm{~N}=60)$ deles. Para ansiedade, a média de pontos foi de $9,7(d p=4,8)$, mediana de 10 , mínimo de 0 e máximo de 20. Quanto à depressão, a média de pontos foi de 7,6 (dp =5,2), mediana de 7, valor mínimo de 0 e máximo de 18. A medicação psicotrópica não era utilizada pela maioria dos pacientes $(61,6 \%, n=77)$; entre os que refe- riram utilizar, o maior número foi de pacientes que faziam uso de antidepressivos $(16,8 \%, n=21)$. Os valores de correlação entre ansiedade, depressão e dor indicaram correlação estatística significante entre os escores de ansiedade e depressão com a escala de dor, tanto na análise geral como na dor de tipo neuropática e mista. Na análise geral, referente ao total de casos estudados, conforme os escores de ansiedade e de depressão aumentaram, aumentou a intensidade da dor, respectivamente, $r=0,41(p<0,001)$ e $r=0,36(p<0,001)$.

$\mathrm{Na}$ análise de regressão linear, verificou-se que a intensidade da dor aumentou $\beta=0,19(p<0,001)$ a cada ponto de ansiedade, e $\beta=0,17(p<0,001)$ a cada ponto de depressão. $\mathrm{Na}$ dor nociceptiva não houve correlação significante para ansiedade $(\beta=0,08 ; p=0,489)$ e depressão $(\beta=0,03 ; p=$ 0790). Quanto ao grupo com dor neuropática, houve aumento de $\beta=0,27(p=0,011)$ na escala de dor a cada ponto do escore de ansiedade e de $\beta=0,30(p<0,001)$ a cada ponto de depressão. Em relação aos pacientes com dor mista, houve aumento de $\beta=0,15$ ( $p=0,001)$ na escala de dor a cada ponto do escore de ansiedade e de $\beta=0,12(p=0,009)$ a cada ponto de depressão. Observou-se correlação significante entre sintomas de depressão e ansiedade $(p=0,001)$.

Renda e religião foram as categorias sociodemográficas que apresentaram associações significantes com a dor. Pacientes que recebiam até dois salários-mínimos mensais tiveram probabilidade de $74 \%$ de apresentarem dor intensa; para pacientes com renda entre três e quatro salários-mínimos e acima de cinco salários-mínimos, essa proporção foi de, respectivamente, $66,7 \%$ e $37,5 \%$ ( $p=0,008)$. Observou-se variação da intensidade da dor conforme as três faixas de renda, notando-se dor intensa prevalente nas faixas mais baixas e dor moderada prevalente na faixa acima de cinco salários-mínimos.

Em relação às variáveis independentes clínicas, verificouse que os fatores tempo de dor, ansiedade e depressão apresentaram associação significante com intensidade de dor. Quanto ao tempo de dor, pacientes que relataram dor entre 24 e 47 meses e 48 e 84 meses (segundo e terceiro quartis) apresentaram probabilidade maior de dor intensa quando comparados a pacientes nos outros dois quartis $(p=0,036)$. Pacientes com nível de ansiedade possível ou provável tiveram maior probabilidade de apresentar dor intensa quando comparados aos pacientes com nível de ansiedade improvável $(73,2 \%$ versus $46,5 \% ; p=0,003)$. O mesmo foi observado para depressão: pacientes com nível de depressão possível ou provável tenderam a apresentar maior intensidade de dor quando comparados a pacientes com nível de depressão improvável (78,3\% versus 50,8\%; $p=0,01)$. A variável funcionalidade não apresentou correlação significante.

Por meio da análise de regressão logística univariada para variáveis sociodemográficas, verificou-se que quanto menor a renda, maior a chance de um paciente apresentar dor intensa. Pacientes que ganhavam até dois salários apre- 
sentaram chance de $O R=4,74(p=0,003)$ e pacientes entre três e quatro salários apresentaram chance de $\mathrm{OR}=3,33$ $(p=0,020)$ para dor intensa, se comparados aos que recebiam mais de cinco salários. Ressalta-se, ainda, a existência de um fator protetor para dor intensa em pacientes nas categorias outras religiões $(O R=0,24 ; p=0,042)$ e sem religião $(O R=0,22 ; p=0,050)$, quando comparados aos católicos.

Na regressão logística univariada para variáveis independentes clínicas, verificou-se, quanto ao tempo de dor, que pacientes com maior chance de apresentarem dor intensa estavam entre o segundo $(O R=4,33 ; p=0,009)$ e terceiro $(\mathrm{OR}=3,75 ; \mathrm{p}=0,024)$ quartis, comparados aos pacientes que relataram dor entre três e dezoito meses.

Notou-se ainda que pacientes com níveis de ansiedade e depressão possível ou provável apresentaram chance de OR $=3,14(p=0,004)$ e $O R=3,51(p=0,002)$, respectivamente, de apresentarem dor intensa, quando comparados a pacientes com níveis improváveis para ansiedade e depressão.

\section{DISCUSSÃO}

Os resultados obtidos no presente estudo mostraram alta prevalência de dor intensa, alcançando $64 \%$ do total da amostra. Comparando-se com outros estudos sobre dor crônica em serviços brasileiros ${ }^{11,12}$, verifica-se que a dor crônica intensa apresenta variabilidade de $32 \%$ a $73,4 \%$. Considerando-se que a dor intensa é estimada em $11 \%$ de adultos portadores de dor crônica ${ }^{5}$, compreende-se que os resultados de alta prevalência de dor crônica intensa em centros de dor são coerentes, dada a especificidade dos serviços e da população que os procura.

Dois estudos brasileiros sobre sintomas de ansiedade e depressão em comorbidade com dor crônica foram realizados em centros de dor ${ }^{11,12}$, identificando relação direta entre os sintomas pesquisados e intensidade de dor. Com amostra de 400 pacientes com dor crônica ${ }^{11}$, uma das pesquisas teve como objetivo analisar a associação dos sintomas psiquiátricos com dor crônica e o impacto na qualidade de vida. Os resultados apontaram que $21 \%$ dos pacientes não apresentaram sintomas depressivo-ansiosos, $7 \%$ apresentaram sintomas depressivos, $18 \%$ apresentaram sintomas ansiosos e 54\% apresentaram ambos. Observou-se ainda que, entre os pacientes com dores mais intensas, 70,4\% apresentaram sintomas ansiosos e 62,3\% apresentaram sintomas depressivos; nos casos de dor leve e moderada a taxa era menor: 59,5\% apresentaram ansiedade e 43,9\%, depressão. Os pacientes com comorbidade psiquiátrica apontaram maiores índices de intensidade de dor e piora em todos os domínios de qualidade de vida avaliada por escala específica". No outro estudo, realizado com 54 pacientes ambulatoriais portadores de neuropatia periférica ${ }^{12}$, 25,9\% não apresentaram sintomas, 5,6\% apresentaram sintomas depressivos, 22,2\% apresentaram sintomas ansiosos e 46,3\% apresentaram ambos os sintomas.

O presente estudo avaliou os sintomas depressivos e ansiosos na população estudada em relação aos diferentes tipos de dor, tendo como desfecho a magnitude de dor. Sua possível contribuição à prática clínica reside na discriminação dos diferentes tipos e intensidades de dor em relação à ocorrência dos sintomas psiquiátricos, podendo auxiliar no melhor manejo desses pacientes.

A proporção de pacientes que apresentavam sintomas psiquiátricos combinados foi elevada nos três estudos brasileiros (contando o presente estudo), o que sugere correlação significante entre essas categorias de sintomas. No presente estudo a correlação é significante $(p=0,001)$. Por conta disso, essa forte correlação divide a opinião dos pesquisadores, havendo alguns que defendem que a HAD seja avaliada na íntegra com a soma dos 14 itens, resultando em uma única medida de morbidade. Outros estudiosos enfatizam a utilização das duas subescalas, pois são úteis ao raciocínio clínico os construtos pesquisados de forma separada, tendo em vista a devida compreensão e encaminhamento dos casos $^{3,13}$.

A escala HAD é indicada e utilizada como bom instrumento para triagem de sintomas psiquiátricos e tem a vantagem de não questionar sintomas físicos que podem confundir ou distorcer os resultados no caso de pacientes com dor crônica. A dor crônica, no entanto, pode suscitar com maior frequência os três conceitos centrais pesquisados nos itens da HAD-A, ou seja, tensão, medo e pânico, e isso justificaria a maior presença de sintomas ansiosos nos resultados pesquisados com essa escala, uma vez que a dor crônica flutua em termos de intensidade e transcorre com episódios de reagudização em meio a períodos relativamente mais calmos. Por outro lado, percebe-se maior concentração dos itens da HAD -D em torno de apenas um conceito, relativo à anedonia, que se refere mais amplamente a vários fatores da vida do paciente. Deve-se, por último, considerar a correlação entre depressão e ansiedade, pois as duas aparecem juntas em quase metade das amostras dos estudos acima comparados ${ }^{14}$.

A relação entre transtornos psiquiátricos e dor crônica tem sido estudada com maior frequência nos casos de depressão, como categoria diagnóstica, e vários modelos foram desenvolvidos na tentativa de compreender essa relação, no entanto de todo modo se sabe que dor e depressão são fenômenos que se retroalimentam. A patogênese refere mecanismos de neurotransmissores comuns e áreas cerebrais comuns envolvidas nos dois fenômenos. Isso não é suficiente ainda para explicar também a questão temporal, ou seja, qual fenômeno se estabelece primeiro em cada caso.

Com relação à intensidade da dor, no presente estudo os sintomas de ansiedade e depressão apresentaram correlação positiva. A regressão linear foi de 0,17 com $O R=3,51$ $(p=0,002)$ para depressão e de 0,19 com $O R=3,14(p=$ $0,004)$ para ansiedade, evidenciando a interferência dos sin- 
tomas psiquiátricos estudados na flutuação da intensidade de dor, havendo interferência levemente maior do quadro depressivo em termos de chances, no entanto não em termos de aumento da intensidade da dor, quesito no qual a ansiedade mostrou correlação discretamente maior.

Dores dos tipos neuropática e mista apresentaram associação significante $(p<0,001)$ entre os escores de ansiedade e depressão com dor intensa. A dor nociceptiva não apresentou associação estatística significante. Pode-se supor que as características da dor nociceptiva estejam relacionadas a esse resultado, pelo fato de essa dor responder bem a analgésicos comuns e também pelo fato de a presença da lesão tecidual informar ao paciente uma possível relação de causalidade.

A dor neuropática apontou, no presente estudo, aumento da intensidade de dor a cada ponto de depressão $(0,30)$ e a cada ponto de ansiedade $(0,27)$. Estudo com pacientes com esse tipo de dor ${ }^{3}$ apresentou resultados semelhantes, com aumento de intensidade da dor de 0,25 e 0,27 para ansiedade e depressão, respectivamente. Essas correlações, embora moderadas, apontam para a necessidade de aprofundamento em novos estudos que verifiquem o comportamento dos transtornos psiquiátricos nos diferentes tipos de dor para melhor abordagem clínica e terapêutica.

Quanto ao tempo de dor, os pacientes que relataram dor entre 19 e 36 meses e 37 e 84 meses (segundo e terceiro quartis) apresentaram probabilidade maior de dor intensa quando comparados a pacientes nos outros dois quartis -3 a 18 meses e 85 a 480 meses $(p=0,036)$. A regressão logística univariada apontou $O R=4,33(p=0,009)$ e $O R=3,75(p=$ $0,024)$ para o segundo e terceiro quartis, respectivamente. Nota-se, no entanto, que $39,2 \%$ da amostra ( $N=49)$ foram pacientes que têm dor crônica há seis anos ou mais; desse total, 65,3\% referiram dor intensa, o que põe em relevo desafios quanto à adesão ao tratamento e à compreensão dos comportamentos dolorosos de pacientes que passam a não conceber suas vidas sem dor ${ }^{8}$.

A literatura refere maior prevalência de dor crônica em idosos, porém apenas um estudo apresentou correlação positiva entre tempo e intensidade de dor, e somente para o sexo feminino 7 . No presente estudo, a dor mais intensa foi relacionada aos quartis intermediários, de um ano e meio a sete anos, sugerindo que a dor ainda era uma experiência nova nos pacientes do primeiro quartil e que os pacientes com mais tempo de dor e também mais idosos tendiam a buscar uma adaptação ao quadro álgico, embora sofram também com as flutuações da intensidade da dor.

Quanto ao perfil da população atendida no serviço investigado, os pacientes apresentaram as seguintes características sociodemográficas: maioria do sexo feminino, na proporção de 2:1; idades variando de 19 a 92 anos (média de 55,4 - dp 15,6; mediana de 57); escolaridade mais frequente nos níveis fundamental incompleto $(36, \%)$ e médio completo (32,8\%); renda familiar de até quatro salários-míni- mos (80\%); casados ou com união estável (59,2\%); com filhos (83,2\%, mediana de dois filhos, variando de 1 a 11); morando com cônjuges (59,2\%), parentes ou acompanhantes (30,4\%) e apenas 10,4\% morando sozinhos; predominantemente católicos (60\%) ou evangélicos (25,6\%); e a maioria encontravase inativa quanto ao trabalho $(69,6 \%)$.

Com relação à variável dependente aqui investigada, qual seja, a intensidade da dor crônica, os fatores sociodemográficos que apresentaram correlação positiva foram renda e religião.

Com relação à renda, os pacientes com renda de até dois salários-mínimos apresentaram $O R=4,74(p=0,003)$ para dor intensa. A literatura refere associação consistente entre dor crônica e nível socioeconômico baixo, o que envolve renda, escolaridade e situação de desemprego ou afastamento ${ }^{4,15}$. São aspectos importantes a considerar na população de baixa renda a falta de acesso a serviços de saúde e a medicamentos adequados, bem como informações compreensíveis sobre suas morbidades e modos de tratamento.

Com relação à religião, neste estudo as categorias "ausência de religião" e "outras religiões" se comportaram como fatores protetores para dor intensa. Esse resultado merece maiores investigações, pois as pesquisas discutem a função positiva da religiosidade/espiritualidade como forma de lidar (coping) com a dor e também há pesquisas que indicam aumento do limiar da dor, redução dos níveis de hormônios adenocorticotrófico e cortisol séricos, diminuição da pressão arterial sistólica, frequência cardíaca e respiratória; indicam, ainda, que a ativação do córtex pré-frontal efetuada por meio de práticas religiosas melhora a interatividade do sistema hipotálamo-pituitária-adrenal e também a elevação dos neurotransmissores envolvidos na dor, quais sejam, GABA, serotonina e dopamina ${ }^{16}$.

Entre as limitações deste estudo, observa-se que a exclusão dos analfabetos nos critérios de amostragem acabou por não abarcar um grupo que, por falta de acesso a informações e tratamento adequados, representa importante desafio, pois esse grupo tenderia a aumentar, na amostragem, o número de pacientes com dor intensa.

A amostra de conveniência utilizada resultou em grupos muito heterogêneos quanto aos tipos de dor. Embora os resultados apontem para uma diferenciação entre sintomas depressivos e ansiosos quanto à magnitude de dor para dor mista e neuropática, uma amostra maior poderia evidenciar de forma mais substancial essas diferenças, que não se apresentaram para dor nociceptiva.

Com relação às questões metodológicas nas investigações das comorbidades aqui pesquisadas, observou-se a necessidade de padronização dos instrumentos e da forma de coleta de dados para melhor conhecimento do paciente com dor crônica em função de:

- alta seletividade das amostras trabalhadas, o que reduz bastante o número de participantes, portanto os achados são parciais e não generalizáveis ${ }^{10}$; 
- $\quad$ variação dos instrumentos utilizados na detecção de transtornos psiquiátricos, ou para triagem de sintomas, de modo que alguns estudos trabalham com sintoma em uma abordagem dimensional e outros com categorias diagnósticas ${ }^{9}$.

Com relação à prática clínica, a HAD apresenta-se como facilitador na triagem de casos de dor crônica que requerem diagnóstico psiquiátrico de depressão e ansiedade detalhado com vistas ao tratamento. No entanto, é importante frisar a necessidade de padronização dos instrumentos diagnósticos ou o respeito aos escores já sugeridos em pesquisas publicadas.

\section{CONCLUSÃO}

Há elevada prevalência de sintomas depressivos e ansiosos em pacientes com dor crônica, e correlação significante com a intensidade e os tipos de dor neuropática e mista, o que requer investigação diagnóstica mais aprofundada para abordagem simultânea dos transtornos psiquiátricos e do quadro álgico.

\section{CONTRIBUIÇÕES INDIVIDUAIS}

Ricardo Cardoso Pinheiro - Contribuiu no desenho do estudo, planejamento, execução, análise dos dados, elaboração do texto.

Ricardo Riyoiti Uchida - Contribuiu na análise dos dados e elaboração do texto.

Lígia Andrade da Silva Telles Mathias - Contribuiu no desenho do estudo, planejamento e análise dos dados.

Marcelo Vaz Perez - Contribuiu no desenho do estudo, planejamento e análise dos dados.

Quirino Cordeiro - Contribuiu no desenho do estudo, planejamento, análise dos dados e elaboração do texto.

\section{CONFLITOS DE INTERESSE}

Os autores declaram não haver conflitos de interesse.

\section{AGRADECIMENTOS}

O autor Ricardo Cardoso Pinheiro agradece a Coordenação de Aperfeiçoamento de Pessoal de Nível Superior/Programa de Suporte à Pós-Graduação de Instituições de Ensino Particulares (Capes/Prosup), pela bolsa de mestrado.

\section{REFERÊNCIAS}

1. Guertzenstein EZ. Transtornos psiquiátricos associados à dor. In: Teixeira MJ. Dor: manual para o clínico. São Paulo: Atheneu; 2006. p. 375-87.

2. Aguiar RW, Caleffi L. Dor crônica. In: Fráguas Jr R, Figueiró JAB, organizadores. Depressões em medicina interna e em outras condições médicas: depressões secundárias. São Paulo: Atheneu; 2000. p. 407-18.

3. Brasil. Ministério da Saúde. Secretaria de Atenção à Saúde. Portaria n 1.083, de 02 de outubro de 2012. Protocolo clínico e diretrizes terapêuticas da dor crônica.

4. Van Hecke O, Torrance N, Smith H. Chronic pain epidemiology and its clinical relevance. Br J Anaesth. 2013;111(1):13-8.

5. Harstall C. How prevalent is chronic pain? Pain Clinical Updates. 2003;11(2):1-4.

6. Ruviaro LF, Filippin LI. Prevalência de dor crônica em uma Unidade Básica de Saúde de cidade de médio porte. Rev Dor (São Paulo). 2012;13(2):128-31.

7. Forni JE, Martins MRI, Rocha CEDA, Foss MHD, Dias LC, Santos Jr R, et al. Perfil sociodemográfico de uma coorte de pacientes encaminhados a uma Clínica de Dor. Rev Dor. 2012;13(2):147-51.

8. Dersh J, Polatin PB, Gatchel RJ. Chronic pain and psychopathology: research findings and theoretical considerations. Psychosom Med. 2002;64(5):773-86.

9. Banks SM, Kerns RD. Explaining high rates of depression in chronic pain: a diathesis-stress framework. Psychol Bull. 1996;119(1):95-110.

10. McWilliams LA, Cox BJ, Enns MW. Mood and anxiety disorders associated with chronic pain: an examination in a nationally representative sample. Pain. 2003;106(1-2):127-33.

11. Brasil ISPS, Pondé MP. Sintomas ansiosos e depressivos e sua correlação com intensidade da dor em pacientes com neuropatia periférica. Rev Psiquiatr RS. 2009;31(1):24-31.

12. Castro MMC, Quarantini LC, Daltro C, Pires-Caldas M, Koenen KC, Kraychete DC, et al. Comorbidade de sintomas ansiosos e depressivos em pacientes com dor crônica e o impacto sobre a qualidade de vida. Rev Psiq Clín. 2011;38(4):126-9.

13. Marcolino JAM, Mathias LAST, Piccinini Filho L, Guaratini AA, Suzuki FM, Alli LAC. Escala Hospitalar de Ansiedade e Depressão: estudo da validade de critério e da confiabilidade com pacientes no pré-operatório. Rev Bras Anestesiol. 2007;57(1):52-62.

14. Botega NJ, Bio MR, Zomignani MA, Garcia Jr C, Pereira WAB. Transtornos de humor em enfermaria de clínica médica e validação de escala de medida (HAD) de ansiedade e depressão. Rev Saúde Pública. 1995;29(5):355-63.

15. Blyth FM, March LM, Brnabic AJM, Jorm LR, Williamson M, Cousins MJ. Chronic pain in Australia: a prevalence study. Pain. 2001;89(2-3):127-34.

16. Lago-Rizzardi CD, Teixeira MJ, Siqueira SRDT. Espiritualidade e religiosidade no enfrentamento da dor. 0 Mundo da Saúde, São Paul0. 2010:34(4):483-7. 